\title{
Alteraciones Motoras Esofágicas en Pacientes con Síndrome de Sjögren. Estudio de Casos y Controles
}

\author{
Esophageal Motility Disorders in Patients with Primary Sjogren's Syndrome. \\ Study of Cases and Controls
}

\author{
Carlos Manterola ${ }^{*, * *, * * * ;}$; Tamara Otzen ${ }^{* *, * * * * *}$ \& Grupo MINCIR.
}

MANTEROLA, C.; OTZEN, T. \& GRUPO MINCIR. Alteraciones motoras esofágicas en pacientes con síndrome de Sjögren. Estudio de casos y controles. Int. J. Morphol., 34(1):285-290, 2016.

RESUMEN: El síndrome de Sjögren primario (SSp), afecta diversos sistemas, como el digestivo; destacando la dificultad deglutoria del bolo alimentario. El objetivo de este estudio es describir la función y motilidad esofágica de pacientes con SSpy compararla con sujetos sanos. Estudio de casos y controles, realizado en Clínica Mayor de Temuco (2004-2009). Se estudiaron pacientes con $\mathrm{SSp}$, con disfagia permanente, sin límite de edad ni sexo (casos); y sujetos asintomáticos digestivos (controles); emparejados pr edad y sexo. Se estudiaron con manometría esofágica estacionaria. Se determinó presión de reposo (PR) y longitud (L) del esfínter esof ágico inferior (EEI) y superior (EES); amplitud (A) y duración (D) de las ondas peristálticas desencadenadas con degluciones secas byúmedas en los tres tercios del cuerpo esofágico (S, M e I), y aparición de ondas terciarias (OT). Se estimó una muestra 20 casos y 40 controles. Se aplicó estadística descriptiva, $\mathrm{Chi}^{2}$ de Pearson o exacto de Fischer para variables categóricas; y Prueba t para variables continuas. La PREEI fue menor en los casos ( 1 1,8 $\pm 0,7 \mathrm{mmHg}$ vs. $14,8 \pm 0,5 \mathrm{mmHg}$ respectivamente). Se verificaron diferencias estadísticamente significativas en laAS, DM, DI y OT tras las degluciones secas; y en AS, AM, AI, DM, DI y OT, tras las degluciones húmedas. No hubo variaciones en la PREES ni LEES entre casos y controles. Se verificaron alteraciones motoras esofágicas y baja PREEI en pacient con $\mathrm{SSp}$, respecto de un grupo de controles.

PALABRAS CLAVE: Síndrome de Sjogren; Enfermedades esofágicas; Manometría esofágica; Estudios de casos y controles.

\section{INTRODUCCIÓN}

El síndrome de Sjögren primario (SSp), es una entidad nosológica de origen autoinmune, progresión lenta, que afecta 20 veces más a mujeres que a hombres (Alamanoset al., 2006; Patel \& Shahane, 2014) y que evoluciona con potencial afectación de diversos órganos y sistemas, como el tracto respiratorio (Kreider \& Highland, 2014), endocrino (Mavragani et al., 2012), renal (Evans et al., 2015), digestivo (Mavragani \& Moutsopoulos, 2014), etc.

La incidencia reportada para SSp en población griega es de 5,3 per 100,000 (IC 95\% 4,5-6,1) (Alamanos et al.). En población latina, existe poca información, sólo se menciona a que podría afectar al $3 \%$ de la población (Ramos-Casals et al., 2003). La prevalencia es inconexa, debido a lo poco definido de sus manifestaciones clínicas y dada la diversidad de criterios de clasificación utilizados por los diversos grupos que publican respecto del tema.
Respecto de las manifestaciones digestivas, una de las más frecuentes y relevantes se relacionan con la dificultad deglutoria del bolo alimentario y su ulterior progresión a lo largo del esófago (Rogus-Pulia \& Logemann, 201; Eberth). La disfagia es un síntoma común en los pacientes con SSp, y se debe a la reducción en la producción de saliva, y probablemente a alteraciones en la motilidad esofágica (Mavragani \& Moutsopoulos).

Sin embargo, a pesar de la frecuencia de aparición de disfagia, los estudios de función motora del esófago son escasos (Ramírez-Mata et al., 1976; Tsianos et al., 1986; Kjellén et al., 1986; Grande et al., 1993; Palma et al., 1994; Anselmino et al., 1997; Rosztóczy et al., 2001; Volter et al., 2004; Türk et al., 2005) y los resultados dispares; desde aquellos que encuentran alteraciones significativas (Rosztóczy et al.), los que encontraron alteraciones parciales (Anselmino

* Departamento de Cirugía y CEMyQ, Universidad de La Frontera, Temuco, Chile.

** Centro de Investigaciones Biomédicas, Universidad Autónoma de Chile, Temuco, Chile.

${ }^{* * *}$ Programa Doctorado en Ciencias Médicas, Universidad de La Frontera, Temuco, Chile.

${ }^{* * * *}$ Fellow Research, Universidad Científica del Sur, Lima, Perú. 
et al.), hasta aquellos que no lograron evidenciar un patrón motor consistente (Grande et al.).

El objetivo de este estudio fur describir la función y motilidad esofágica de pacientes con SSp y compararla con sujetos sanos.

\section{MATERIAL Y MÉTODO}

Tipo de estudio: Estudio de casos y controles.

Escenario: El estudio fue realizado en Clínica Mayor de Temuco (Red de Clínicas Regionales), entre los años 2004 y 2009 .

Población: Pacientes portadores de síndrome de Sjögren primario (SSp), diagnosticados de acuerdo a los criterios del Consenso del grupo Americano-Europeo (AECG); que consisten en la presencia de: xerostomía clínica, queratoconjuntivitis seca demostrada mediante prueba de Schirmer; y presencia de anticuerpos anti-Ro/SSA y antiLa/SSB (Vitali et al., 2002; Langegger et al., 2007); sin límite de edad ni sexo, con disfagia permanente, que fueron derivados a estudio funcional esofágico (a continuación se denominarán como "casos"). Voluntarios sanos, asintomáticos digestivos, que no ingerían medicamentos que pudiesen interferir en la motilidad esofágica (a continuación se denominarán como "controles"). Estos, fueron emparejados por edad y sexo con los casos (Thbla I). Los casos fueron hospitalarios prevalentes e incidentes; y los controles, poblacionales e incidentes.

Tabla I. Emparejamiento entre Casos y Controles.

\begin{tabular}{lcccc}
\hline \multirow{2}{*}{ Variable } & Casos & Controles & \multirow{2}{*}{$\mathbf{p}$} \\
\cline { 2 - 4 } & $(\mathbf{n}=\mathbf{2 0})$ & $(\mathbf{n}=\mathbf{4 0})$ & \\
\hline \multicolumn{2}{l}{ Edad (años) } & $51,1 \pm 1,5$ & $50,9 \pm 0,9$ & 0,8998 \\
Sexo & Femenino & 94,7 & 95,0 & \multirow{2}{*}{1,0000} \\
& Masculino & 5,3 & 5,0 & \\
\hline
\end{tabular}

Protocolo de estudio: Tanto casos como controles fueron estudiados mediante manometría esofágica según técnica previamente descrita (Manterola et al., 2000; Manterola et al., 2004); pero que de forma sucinta se realizó con sujeto en decúbito supino, introduciendo a través de la nariz y hasta el estómago una sonda de tres canales (M3 Arndorfer Medical Specialties, Milwaukee, Wisconsin ${ }^{\circledR}$ ) con orificios laterales abiertos cada $5 \mathrm{~cm}$ y orientados en sentido circunferencial cada $120^{\circ}$. Los catéteres fueron perfundidos de forma constante mediante una bomba de infusión neumohidráulica capilar tipo Arndorfer, a $0,6 \mathrm{~mL} / \mathrm{min}$ de débito. y conectados a transductores externos y un polígrafo multicanal. Se determinó la presión de reposo (PR) y longitud (L) del esfínter esofágico inferior (EEI) mediante técnica de retirada estacionaria, y con el orificio distal de la sonda situado $5 \mathrm{~cm}$ por encima del magen superior del EEI. Se valoró la amplitud (A) y duración (D) de las ondas peristálticas en los tercios superior (S), medio (M) e inferior (I); desencadenadas tras 10 degluciones secas (saliva disponible) y posteriormente tras 10 degluciones de agua a temperatura ambiente de $5 \mathrm{~mL}$ cada una (en ambas maniobras se dejó transcurrir un lapso de 30 s entre una y otra deglución). Además se determinó la aparición de ondas terciarias (OT). Finalmente, se verificó la PR y Ldel esfínter esofágico superior (EES), mediante técnica similar a la utilizada para el estudio del EEI.

Variables de interés: La variable resultado principal fue el comportamiento de la motilidad del cuerpo esofágico tras degluciones secas y húmedas (ingestión de saliva disponible y $5 \mathrm{cc}$ de agua respectivamente) y los parámetros funcionales de los EEI y EES.

Tamaño de la muestra: Se estimó considerando un inter valo de confianza del $95 \%$, una potencia de $80 \%$, relación casos : controles de 2:1 y una diferencia de medición en la amplitud de las ondas motoras del cuerpo esofágico del 40 $\%$ entre casos y controles. De esta manera, se obtuvo una muestra de 57 sujetos a estudio, 19 casos y 38 controles. La distribución por sexo y edad de casos y controles se aprecia en la Tabla I.

Plan de análisis: Se realizó un análisis exploratorio de los datos, aplicando estadística descriptiva y posteriormente se aplicó $\mathrm{Chi}^{2}$ de Pearson o prueba exacta de Fischer para variables categóricas; y la Prueba t para variables continuas. Finalmente, se calcularon los odds ratios (OR) para las variables dicotómicas. Los cálculos fueron realizados en programa estadístico STATA/SE 11.1.

Aspectos éticos: Todos los participantes firmaron un consentimiento informado.

\section{RESULTADOS}

La PREEI fue significativamente menor en los casos que en los controles $(11,8 \pm 0,7 \mathrm{mmHg}$ vs. $14,8 \pm 0,5 \mathrm{mmHg}$ respectivamente $[p=0,0013])$. Por otra parte, en la LEEI no se verificaron diferencias estadísticamente significativas entre casos y controles $(3,5 \pm 0,2 \mathrm{~cm}$ vs. $3,6 \pm 0,1 \mathrm{~cm}$, respectivamente $[\mathrm{p}=0,4720])$ (Figs. 1 y 2 ).

Respecto del cuerpo esofágico, se logró evidenciar 


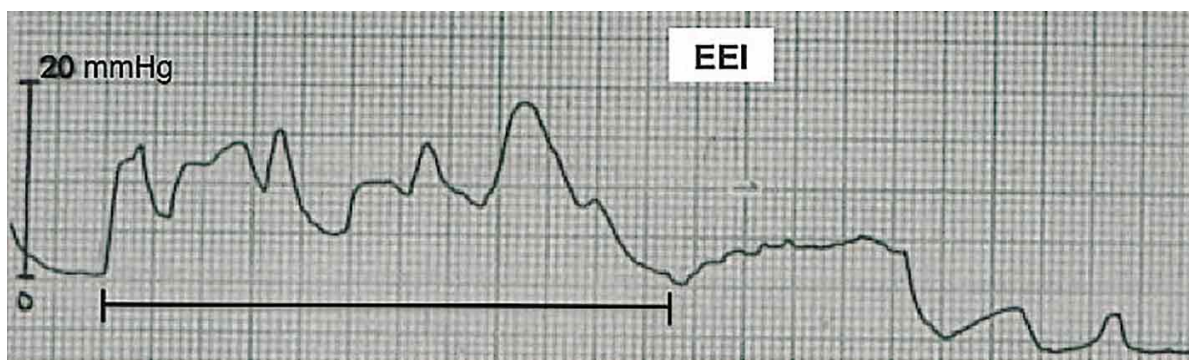

Fig. 1. Trazado manométrico, en el que se aprecia la morfología, presión de reposo y longitud del EEI de un sujeto del grupo control.

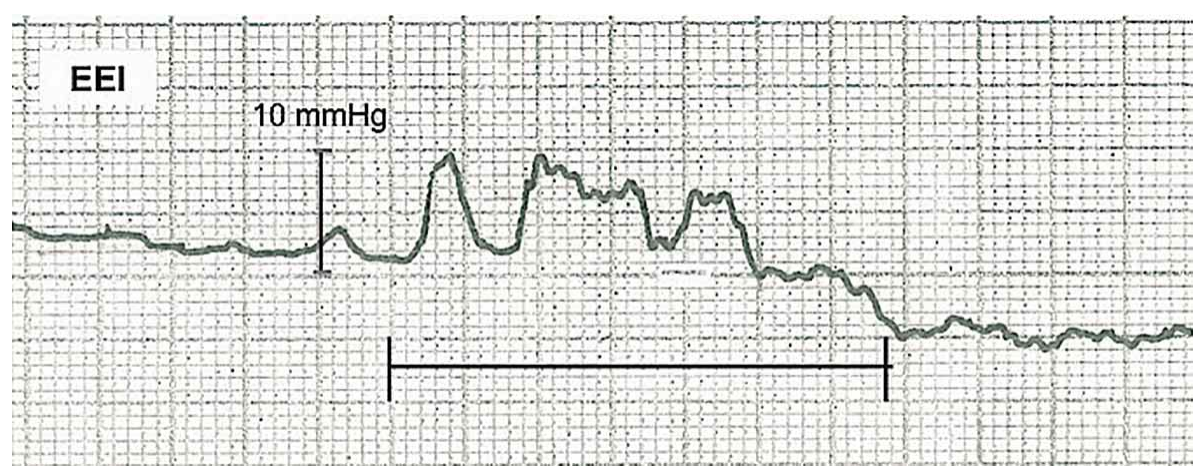

Fig. 2. Trazado manométrico, en el que se aprecia la morfología presión de reposo y longitud del EEI de un paciente con SSp (caso).

diferencias estadísticamente significativas en las variables AS, DM, DI y OT, tras las degluciones secas (Tabla II); y, diferencias estadísticamente significativas en las variables
AS, AM, AI, DM, DI y OT, tras las degluciones húmedas (Tabla III). Un ejemplo de esto, se puede observar en las Figuras $3 \mathrm{~A}$ y $3 \mathrm{~B}$.

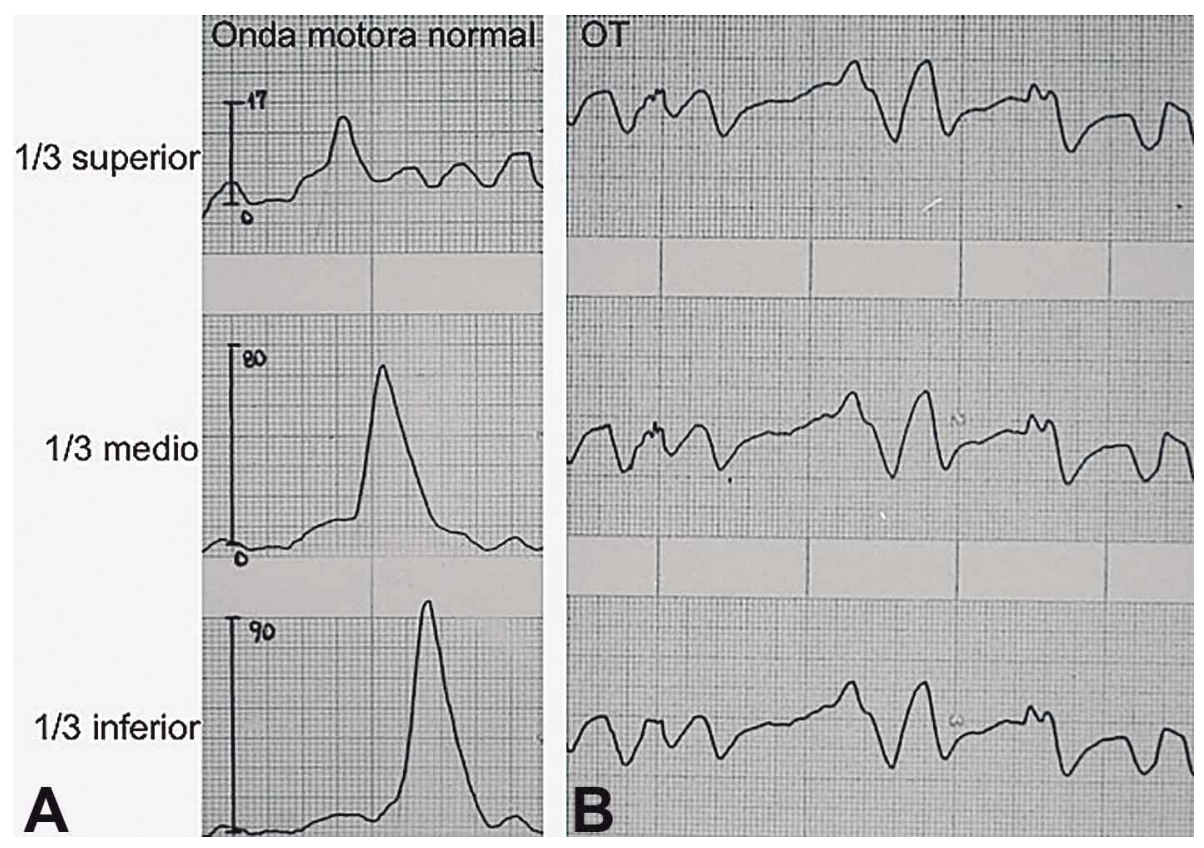

Fig. 3. A) Trazado manométrico, en el que se aprecia la morfología de las ondas motoras (amplitud y duración) del cuerpo esofágico en sus tercios superior, medio e inferior; en un sujeto del grupo control. B) Trazado manométrico, en el que se aprecia la morfología de las ondas terciarias o no transmitidas de un paciente con SSp (caso). 
Tabla II. Características manométricas del cuerpo esofágico tras degluciones secas.

\begin{tabular}{lccc}
\hline \multirow{2}{*}{ Parámetro } & Casos & Controles & p \\
\cline { 2 - 3 } & $(\mathbf{n = 2 0})$ & $\mathbf{( n = 4 0 )}$ & \\
\hline AS (mmHg) & $24,1 \pm 1,0$ & $28,9 \pm 1,5$ & 0,0118 \\
AM (mmHg) & $40,4 \pm 2,5$ & $43,2 \pm 1,5$ & 0,3288 \\
AI (mmHg) & $61,7 \pm 2,8$ & $66,2 \pm 1,5$ & 0,1284 \\
DS (cm) & $3,3 \pm 0,1$ & $3,5 \pm 0,1$ & 0,1352 \\
DM (cm) & $3,6 \pm 0,1$ & $3,9 \pm 0,1$ & 0,0312 \\
DI (cm) & $3,8 \pm 0,1$ & $4,1 \pm 0,1$ & 0,0010 \\
OT $(\%) *$ & $9,5 \pm 2,3$ & $2,3 \pm 0,6$ & 0,0003 \\
\hline
\end{tabular}

*= OR de OT para casos y controles fue de 6,0 (IC de $95 \%$ de 4,1 a 8,0$)$.

Es interesante que el cálculo de OR, permitió demostrar que en el caso de las degluciones secas, el OR fue 6,0 (IC de $95 \%$ de 4,1 a 8,0); y en el caso de las degluciones húmedas, de 7,0 (IC de $95 \%$ de 4,6 a 9,1).

Por otra parte, no se detectaron variaciones significativas en la PREES $(34,3+9,5 \mathrm{mmHg}$ vs. $37,8 \pm 10,1 \mathrm{mmHg}$ $[\mathrm{p}=0,5010])$, ni en la LEES $(3,3 \pm 0,1 \mathrm{~cm}$ vs. $3,5 \pm 0,1 \mathrm{~cm}$, respectivamente $[\mathrm{p}=0,5710])$; entre casos y controles.

Tabla III. Características manométricas del cuerpo esofágico tras degluciones húmedas.

\begin{tabular}{lccc}
\hline \multirow{2}{*}{ Parámetro } & Casos & Controles & p \\
\cline { 2 - 3 } & $(\mathbf{n}=\mathbf{2 0})$ & $(\mathbf{n}=\mathbf{4 0})$ & \\
\hline AS $(\mathrm{mmHg})$ & $22,5 \pm 1,5$ & $35,1 \pm 1,9$ & 0,001 \\
AM $(\mathrm{mmHg})$ & $40,1 \pm 1,9$ & $51,5 \pm 1,9$ & 0,001 \\
AI (mmHg) & $49,5 \pm 2,0$ & $63,4 \pm 2,1$ & 0,001 \\
DS (cm) & $3,3 \pm 0,1$ & $3,5 \pm 0,1$ & 0,1352 \\
DM (cm) & $3,6 \pm 0,1$ & $3,9 \pm 0,1$ & 0,0312 \\
DI (cm) & $3,8 \pm 0,1$ & $4,1 \pm 0,1$ & 0,0010 \\
OT $(\%)$ & $10,5 \pm 2,1$ & $2,3 \pm 0,6$ & 0,0019 \\
\hline
\end{tabular}

$*=$ OR de OT para casos y controles fue de 7,0 (IC de $95 \%$ de 4,6 a 9,1$)$.

\section{DISCUSIÓN}

Este estudio demuestra que los pacientes con SSp tienen alteraciones en algunos de los diferentes parámetros funcionales y motores esofágicos evaluados mediante manometría estacionaria; tanto con degluciones secas como húmedas. El OR de 6,0 y de 7,0; representa que los casos tienen 6 veces más probabilidades de desarrollar OTque los controles tras las degluciones secas; y 7 veces más probabilidades de desarrollar OT que los controles tras las degluciones húmedas.

Al respecto, existe evidencia que sustenta el hecho que en sujetos sanos, existen diferencias de la motilidad según que el estímulo que inicie las ondas sea una deglución seca (saliva disponible) o húmeda (ingestión de una cantidad conocida de agua) (Grande et al.).

Por otro lado, existe evidencia que sustenta el hecho que pacientes con SSp, tienen menor PREEI al compararlos con un grupo de controles; y a nivel del cuerpo esofágico, una reducción de la velocidad peristáltica e incremento de las OT (Rosztóczy et al.).

En contraste con los hallazgos anteriormente descritos; otro estudio, demostró ausencia de anormalidades funcionales del EEI y del EES en sujetos con SSp; así como la motilidad del cuerpo esofágico. Lo único que destaca de este estudio, es la aparición de OT en más del $30 \%$ de las degluciones húmedas, hallazgo que se asoció a la existencia de disfagia en estos casos (Anselmino et al.). Algo similar fue anteriormente descrito, como diferencias menores en la motilidad entre pacientes con SS y un grupo de controles: disminución en la duración de las ondas motoras e incremento de la velocidad de éstas, en especial en el tercio distal del esófago (Kjellén et al.). En otro similar, se evidenció una velocidad de progresión mayor en el tercio distal del cuerpo esofágico y menor duración de las ondas peristálticas en sujetos con SSp y secundario; pero no se realizó una diferenciación entre un subgrupo y el otro (Kjellén et al.).

Sin embargo, también se ha evidenciado anormalidades motoras hasta en el $33 \%$ de sujetos con SS, los que no se asocian con la presencia de disfagia, compromiso extraglandular, ni de anticuerpos circulantes (Palma et al.).

Otro estudio, aporta evidencia contraria, en términos que sólo existiría menor velocidad de progresión de la onda peristáltica en pacientes con SS (Grande et al.); no así alteraciones de la motilidad esofágica; ni siquiera tras las degluciones de saliva, cuyo volumen está disminuido; lo que podría explicarse por la reacción inflamatoria de las glándulas exocrinas del cuerpo esofágico o por una miositis, lo que ya se ha sugerido (Tsianos et al.).

Por otra parte, como todos los casos presentaban disfagia, no hubo posibilidad de comparar las características motoras esofágicas entre casos con y sin disfagia; sin embargo, en un estudio similar, no se evidenció asociación entre estas variables (Grande et al.).

Otro estudio, permitió verificar que los pacientes con SS tenían un $29 \%$ de OTindependiente si se tratara de SSp o secundario; así como un mayor tiempo de exposición ácida del esófago en estos pacientes, en presencia de OT , hecho fundamental, pues el aclaramiento deteriorado (falta de salivación y dismotilidad), los predispone a daño de la mucosa esofágica (Volter et al.). 
En conclusión, no existe un consenso respecto de anomalías funcionales esfinterianas ni de la motilidad del esófago en sujetos con SSp; las que pueden deberse no sólo a la heterogeneidad de las poblaciones estudiadas (no sólo SSp y secundario), sino que a lo inespecífico de las manifestaciones clínicas y diversidad de criterios de clasi- ficación utilizados; al grado de avance de la enfermedad y la posible afectación de otros sistemas que de forma directa o indirecta puedan alterar los mecanismos deglutorios. Por otra parte, llama la atención la falta de estudios de este tipo, a pesar del persistente desacuerdo en los resultados observados.

MANTEROLA, C.; OTZEN, T. \& GRUPO MINCIR. Esophageal motility disorders in patients with primary Sjogren's syndrome. Study of cases and controls. Int. J. Morphol., 34(1):285-290, 2016.

SUMMARY: Primary Sjögren's syndrome (PSS), af fects various systems such as the digestive; highlighting the dif ficulty swallowing food bolus. The objective of this study is to describe the function and in patients with esophageal motility PSS copare them to healthy subjects. Case studies and tests conducted at the clinica MayorTemuco (2004-2009). PSS patients with permanent dysphagia, regardless of age or sex (cases) were studied and digestive asymptomatic subjects (controls); matched for age and sex.We studied these with stationary esophageal manometry. Resting pressure (RP) and length (L) of the lower esophageal sphincter (LES) and upper (UES) was determined; amplitude (A) and duration (D) of the peristaltic waves triggered with dry and wet swallows in the three thirds of the esophageal body (S, M and I), and appearance of tertiary waves (TW)A sample of 20 cases and 40 controls were estimated. Desciptive statistics, Pearson $\mathrm{Chi}^{2}$ or Fisher's exact was applied for categorical variables; and t-test for continuous variables.The PREEI was lower in cases $(11.8 \pm 0.7 \mathrm{mmHg}$ vs. $14.8 \pm 0.5 \mathrm{mmHg}$ respectively). Statistically significant diferences in theAS, DM, DI andTW were verified after dry swallows; and inAS, AM, AI, DM, DI andTW after wet swallows. There were no changes in Prees or LEES between cases and controls. Lower esophageal motor disorders was checked in patients with PSS RPEEI, for a control group.

KEY WORDS: Sjogren's Syndrome; Esophageal diseases; Esophageal manometry; Case-control studies.

\section{REFERENCIAS BIBLIOGRÁFICAS}

Alamanos, Y.; Tsifetaki, N.; Voulgari, P. V.; Venetsanopoulou, A. I.; Siozos, C. \& Drosos, A. A. Epidemiology of primary Sjögren's syndrome in north-west Greece, 1982-2003. Rheumatology (Oxford), 45(2):187-91, 2006.

Anselmino, M.; Zaninotto, G.; Costantini, M.; Ostuni, P, Ianniello, A.; Boccú, C.; Doria,A.; Todesco, S. \&Ancona, E. Esophageal motor function in primary Sjögren's syndrome: correlation with dysphagia and xerostomia. Dig. Dis. Sci., 42(1):113-8, 1997.

Ebert, E. C. Gastrointestinal and hepatic manifestations of Sjogren syndrome. J. Clin. Gastroenterol., 46(1):25-30, 2012.

Evans, R.; Zdebik,A.; Ciurtin, C. \&Walsh, S. B. Renal involvement in primary Sjögren's syndrome. Rheumatology (Oxford), 54(9):1541-8, 2015.

Grande, L.; Lacima, G.; Ros, E.; Font, J. \& Pera, C. Esophageal motor function in primary Sjögren's syndrome. Am. J. Gastroenterol., 88(3):378-81, 1993.

Kjellén, G.; Fransson, S. G.; Lindström, F; Sökjer, H. \& Tibbling, L. Esophageal function, radiography, and dysphagia in Sjögren's syndrome. Dig. Dis. Sci., 31(3):225-9, 1986.

Kreider, M. \& Highland, K. Pulmonary involvement in Sjögren syndrome. Semin. Respir. Crit. Care Med., 35(2):255-64, 2014.

Langegger, C.; Wenger, M.; Duftner, C.; Dejaco, C.; Baldissera, I.;
Moncayo, R. \& Schirmer M. Use of the European preliminary criteria, the Breiman-classification tree and the AmericanEuropean criteria for diagnosis of primary Sjögren's Syndrome in daily practice: a retrospective analysis. Rheumatol. Int., 27(8):699-702, 2007.

Manterola, D. C.; Muñoz, N. S.; Flores, O. P.; Fernández, F. E. \& Capurro, C. M. Estudio de asociación entre magnitud de la hipotonía esfinteriana y trastorno motor del cuerpo esofágico. Rev. Med. Chil., 128(7):721-8, 2000.

Manterola, C.; Barroso, M. S.; Losada, H.; Muñoz, S. \& Vial, M. Prevalence of esophageal disorders in patients with recurrent chest pain. Dis. Esophagus, 17(4):285-91, 2004.

Mavragani, C. P.; Fragoulis, G. E. \& Moutsopoulos, H. M. Endocrine alterations in primary Sjogren's syndrome: an overview. J. Autoimmun., 39(4):354-8, 2012.

Mavragani, C. P., \& Moutsopoulos, H. M. Sjögren syndrome. CMAJ, 186(15):E579-86, 2014.

Palma, R.; Freire,A.; Freitas, J.; Morbey, A.; Costa, T.; Saraiva, F; Queirós, F. \& Carvalhinhos, A. Esophageal motility disorders in patients with Sjögren's syndrome.Dig. Dis. Sci., 39(4):75861, 1994.

Patel, R. \& Shahane,A. The epidemiology of Sjögren's syndrome. Clin. Epidemiol., 6:247-55, 2014. 
Ramirez-Mata, M.; Pena Ancira, F. F. \& Alarcon-Segovia, D. Abnormal esophageal motility in primary Sjögren's syndrome. J. Rheumatol., 3(1):63-9, 1976.

Ramos-Casals, M.; García-Carrasco, M.; Anaya, J. M.; Coll, J.; Cervera, R.; Font, J. \& Ingelmo, M. (Eds.). Síndrome de Sjögren. Barcelona, Masson, 2003.

Rogus-Pulia, N. M. \& Logemann, J. A. Effects of reduced saliva production on swallowing in patients with Sjogren's syndrome. Dysphagia,26 (3):295-303, 2011.

Rosztóczy, A.; Kovács, L.; Wittmann, T.; Lonovics, J. \& Pokorny, G. Manometric assessment of impaired esophageal motor function in primary Sjögren's syndrome.Clin. Exp. Rheumatol., 19(2):147-52, 2001.

Tsianos, E. B.; Vasakos, S.; Drosos, A. A.; Malamou-Mitsi, V. D. \& Moutsopoulos H. M. The gastrointestinal involvement in primary Sjögren's syndrome. Scand. J. Rheumatol. (Suppl., 61):151-5, 1986 .

Türk, T.; Pirildar, T.; Tunç, E.; Bor, S. \& Doganavs, ar gil, E. Manometric assessment of esophageal motility in patients with primary Sjögren's syndrome. Rheumatol. Int., 25(4):246-9, 2005.

Vitali, C.; Bombardieri, S.; Jonsson, R.; Moutsopoulos, H. M.; Alexander, E. L.; Carsons, S. E.; Daniels, T. E.; Fox, P. C.; Fox, R. I.; Kassan, S. S.; Pillemer, S. R.; Talal, N.; Weisman, M. H. \& European Study Group on Classification Criteria for Sjögren's Syndrome. Classification criteria for Sjögren's syndrome: a revised version of the European criteria proposed by the American-European Consensus Group. Ann. Rheum. Dis., 61(6):554-8, 2002.

Volter, F.; Fain, O.; Mathieu, E. \&Thomas, M. Esophageal function and Sjögren's syndrome. Dig. Dis. Sci., 49(2):248-53, 2004.
Dirección para Correspondencia:

Dr. Carlos Manterola, MD, PhD.

Departamento de Cirugía y CEMyQ

Universidad de La Frontera

Temuco

CHILE

Teléfono: 56-45-2325760

Fax: 56-45-2325761

Email: carlos.manterola@ufrontera.cl

Recibido : 12-09-2015

Aceptado: 27-12-2015 